\title{
Compliance Matrix of a Single-Bent Leaf Flexure for a Modal Analysis
}

\author{
Nghia-Huu Nguyen, ${ }^{1}$ Moo-Yeon Lee, ${ }^{2}$ Ji-Soo Kim, ${ }^{1}$ and Dong-Yeon Lee ${ }^{1}$ \\ ${ }^{1}$ School of Mechanical Engineering, Yeungnam University, Gyeongsan 712-749, Republic of Korea \\ ${ }^{2}$ Department of Mechanical Engineering, Dong-A University, Busan 604-714, Republic of Korea \\ Correspondence should be addressed to Dong-Yeon Lee; dylee@ynu.ac.kr
}

Received 22 March 2015; Accepted 4 May 2015

Academic Editor: Chao Tao

Copyright (c) 2015 Nghia-Huu Nguyen et al. This is an open access article distributed under the Creative Commons Attribution License, which permits unrestricted use, distribution, and reproduction in any medium, provided the original work is properly cited.

\begin{abstract}
We present a compliance matrix for a single-bent leaf flexure (SBLF) that shows the relationships between the deformations and the six-axis loads applied to the SBLF. Higher-order beam theory that considers the variable shear and warping effect is considered in bending. The partially restrained warping at the junction between elements is also considered in torsion. The total strain energy is calculated, and the complete compliance matrix is derived by using Castigliano's second theorem. Sensitivity analyses over the compliance elements are performed and verified via finite element analysis (FEA). The results show that the derived compliance elements are in good agreement with FEA, with errors of less than $7.6 \%$. We suggest that theoretical compliance elements considering variable shear and warping in bending and partially restrained warping in torsion give highly accurate design equations representing the compliant mechanism of the SBLF. The present work could be used in a modal analysis of a single-bent leaf flexure.
\end{abstract}

\section{Introduction}

Flexure guides are used in precision engineering, especially in nanoscanner devices, because of their smooth, elastic, and no-friction characteristics. Flexure guides provide short or moderate ranges of travel due to their elastic deformation characteristics [1]. New flexure guide which has large working range and nanoresolution is hot topic in the recent years. Several types of flexure guides have been described in the literature including hinge flexures [2-7] and leaf-spring flexures [8-13]. A single-bent leaf flexure, investigated in this study, is an L-shaped leaf-spring mechanism. The SLBF has remarkable advantages for flexure such as a moderate range of deflection and easily obtained uniform spring material. Many studies have focused on flexure guides. Formulas are derived for the stiffness of a parallel leaf-spring flexure in [8]. A displacement reduction mechanism based on torsion leaf-spring hinges was developed in [9]. An orthoplanar spring design that operates by raising or lowering its platform relative to the base with no rotation is presented in [10]. A planar positioning mechanism with three degrees of freedom using a flexure guide is presented in [11]. New flexures were designed and developed with a large range and compact dimensions, as described in $[1,12,14]$.

The compliance matrix is often used to express the loaddeformation relationship for a linearly elastic system. It has been applied in many previous studies in the precision field $[3,4,15-17]$ to derive the equations of motion for general flexure mechanisms. The compliance matrix for a prismatic beam is presented in [3], but the shear deformation and the warping effect in bending and torsion were not considered. The constant shear deformation is considered in bending, the warping is not included in torsion $[4,15]$. The warping was considered in the torsion theory; however, it was applied to the beams with a fully fixed (restrained) end $[18,19]$. The partially restrained warping in torsion was investigated in [20] and was applied to truck chassis frames. The general higher-order beam theory (HBT) was presented in [21-23], and Levinson [21] proposed an equation for the deflection of a beam that includes the shear and warping in bending analysis. Relationships between Levinson beam theory and classical Euler-Bernoulli theory are presented and applied 




(a)



(b)

FIgURE 1: (a) Schematic diagram of a SBLF. (b) Example of the planar scanner using SBLF.

to examples by Reddy et al. [22]. However, these models are usually applied to the cantilever beam or the simply supported beam models, not to the SBLF. The compliance matrix of a bent beam microsuspension was presented in [24]. The beam model is similar to the SBLF model; however, only the in-plane deformations were derived and the shear deformations were not considered in the bending analysis.

In this study, we analyzed and derived a compliance matrix that expresses the relationship between the deformations and applied loads of the SBLF by using Castigliano's second theorem. The HBT of Levinson is applied in the bending analysis. The partially restrained warping at the junction between elements is introduced, and the full and free warping is also investigated in the torsional analysis. The theoretical results are verified by finite element analysis (FEA) results. The good agreement between the results of the two methods validates the accuracy of the theoretical equations.

\section{Generalized Modeling of the SBLF}

Figure 1(a) shows a model of the SBLF that consists of two leaf flexure elements. One end is fixed, and other end has six components of loading: three forces $\left(F_{x}, F_{y}\right.$, and $\left.F_{z}\right)$ and three moments $\left(T_{x}, M_{y}\right.$, and $\left.M_{z}\right)$. Dimensions $l, b$, and $t$ are the length, width, and thickness, respectively. Under the 6-axis loads, axial, bending, shear, and torsional deformations will occur in both elements of the SBLF. Figure 1(b) shows one example of the SBLF in a planar nanoscanner, which includes four SBLFs symmetrically connected with the four corners of the square moving body to ensure smooth and nonparasitic error motion of the system. In this study, the deformations due to the six components of loading need to be analyzed and determined in the compliance matrix form.

2.1. Derivation of the Total Strain Energy. Castigliano's second theorem was used to find the translational and rotational deformations of the SBLF. Thus, the deformations due to loads are defined by the partial derivative of the total strain energy (SE) with respect to the loads as follows:

$$
\begin{gathered}
\delta_{i}=\frac{\partial U}{\partial F_{i}}, \\
\theta_{i}=\frac{\partial U}{\partial M_{i}},
\end{gathered}
$$

where $U$ is the total strain energy that is stored in the flexure, $\delta_{i}$ is the deflection due to the force $F_{i}$, and $\theta_{i}$ is the rotation due to the moment $M_{i}$. When the six components of loading are applied, the total SE stored in two elements of the SBLF can be classified as the extensional, shear, bending, and torsional deformation energies.

The extensional SE $\left(U_{a}\right)$ in element 1 due to the axial force $F_{x}$ are

$$
U_{a 1 F_{x}}=\int_{0}^{l} \frac{F_{x}^{2}}{2 A E} d x
$$

In element 2 , the extensional SE $\left(U_{a}\right)$ due to the axial force $F_{y}$ is given by

$$
U_{a 2 F_{y}}=\int_{0}^{l} \frac{F_{y}^{2}}{2 A E} d y
$$

where $A=b t$ is the cross-sectional area, and $E$ is the elastic modulus (Young's modulus).

In the bending and shear analyses, the shear deformations due to the transverse loads are usually neglected in EulerBernoulli beam theory (EBT). Accordingly, the EBT can predict the deflection accurately only for a thin beam (ratio $l / b>20)$. Timoshenko beam theory considers the additional constant shear deformation. Thus, TBT shows more precise analysis results than EBT. However, for thick beams $(l / b<20)$, the higher-order beam theory that considers the variable shear deformation and the warping in bending should be applied. In the HBT of Levinson, the variable shear deformation and warping in bending are considered and the 
deflection is described in [21]. Therefore, the shear SE $\left(U_{s}\right)$ in element 1 due to the shear forces $F_{y}, F_{z}$ is given by

$$
\begin{aligned}
U_{s 1 F y} & =\int_{0}^{l} \frac{F_{y}{ }^{2} l^{2}}{4 E I_{z}}(1+\nu)\left(\frac{t^{2}}{l^{2}}\right)\left(\frac{x}{l}\right) d x, \\
U_{s 1 F z} & =\int_{0}^{l} \frac{F_{z}^{2} l^{2}}{4 E I_{y}}(1+\nu)\left(\frac{b^{2}}{l^{2}}\right)\left(\frac{x}{l}\right) d x,
\end{aligned}
$$

where $v$ is Poisson's ratio. In element 2, the shear SE $\left(U_{s}\right)$ due to the shear forces $F_{x}, F_{z}$ is given by

$$
\begin{aligned}
U_{s 2 F x} & =\int_{0}^{l} \frac{F_{x}^{2} l^{2}}{4 E I_{z}}(1+v)\left(\frac{t^{2}}{l^{2}}\right)\left(\frac{y}{l}\right) d y ; \\
U_{s 2 F z} & =\int_{0}^{l} \frac{F_{z}^{2} l^{2}}{4 E I_{y}}(1+v)\left(\frac{b^{2}}{l^{2}}\right)\left(\frac{y}{l}\right) d y .
\end{aligned}
$$

The bending SE $\left(U_{b}\right)$ in element 1 due to $F_{z}, F_{y}, M_{y}$, and $M_{z}$ is given by

$$
U_{b 1}=\int_{0}^{l} \frac{\left(M_{y}-F_{z} x\right)^{2}}{2 E I_{y}} d x+\frac{\left(M_{z}+F_{y} x\right)^{2}}{2 E I_{z}} d x .
$$

In element 2 , the bending $\mathrm{SE}\left(U_{b}\right)$ due to $F_{y}, F_{x}, F_{z}, T_{x}$, and $M_{z}$ is given by

$$
U_{b 2}=\int_{0}^{l} \frac{\left(M_{z}+F_{y} l+F_{x} y\right)^{2}}{2 E I_{z}} d y+\frac{\left(T_{x}-F_{z} y\right)^{2}}{2 E I_{x}} d y,
$$

where $I_{x}=I_{y}=t b^{3} / 12$ and $I_{z}=b t^{3} / 12$ is the inertia moment about the $x, y$, and $z$ axes, respectively.

A partially restrained warping torsion analysis at the joint between elements 1 and 2 is presented in [25]. The warping restraint factor $(K)$ is adopted to represent the degree of warping restraint. In the present study, the torsional SE $\left(U_{t}\right)$ was defined in element 1 due to moment $T_{x}$ as follows [25]:

$$
U_{t 1}=\frac{1}{2} E C_{w} \int_{0}^{l}\left(\theta_{x}^{\prime \prime}\right)^{2} d x+\frac{1}{2} G J_{x} \int_{0}^{l}\left(\theta_{x}^{\prime}\right)^{2} d x,
$$

where

$$
\begin{aligned}
\theta_{x} & =\frac{T_{x}}{\alpha G J_{x}}(\alpha x \\
& +(1-K)(-\sinh \alpha x-\tanh \alpha l+\tanh \alpha l \cosh \alpha x)) .
\end{aligned}
$$

In element 2, the torsional SE $\left(U_{t}\right)$ due to moments $F_{z} l$ and $M_{y}$ is as follows:

$$
U_{t 2}=\frac{1}{2} E C_{w} \int_{-l}^{0}\left(\theta_{y}^{\prime \prime}\right)^{2} d y+\frac{1}{2} G J_{y} \int_{-l}^{0}\left(\theta_{y}^{\prime}\right)^{2} d y,
$$

where

$$
\begin{aligned}
\theta_{y} & =\frac{M_{y c}}{\alpha G J_{y}}[\alpha y-\sinh \alpha y \\
& -\left(K \tanh \alpha l-(1-K)\left(\frac{1-\cosh \alpha l}{\sinh \alpha l}\right)\right) \cosh \alpha y \\
& \left.+\left(K \tanh \alpha l-(1-K)\left(\frac{1-\cosh \alpha l}{\sinh \alpha l}\right)\right)\right],
\end{aligned}
$$

where $M_{y c}=M_{y}-F_{z} l$.

The torsion constant $J_{x}$ of a bar with a rectangular cross section can be determined by Equation (161) in [26]. Therefore,

$$
\begin{aligned}
& J_{x}=\frac{b t^{3}}{3}\left[1-0.63 \frac{t}{b}\right], \\
& J_{x}=J_{y} .
\end{aligned}
$$

Warping constant $C_{w}$ with respect to the shear center is defined by Equation (7.43) in [27] as the warping moment of inertia:

$$
C_{w}=\int \omega^{2} d A=\frac{(b t)^{3}}{144}
$$

where $\omega$ is the warping function and $\alpha=\sqrt{G J_{y} / E C_{w}}$ is the torsion-bending constant $(m)$.

$K$, the warping restraint factor, is introduced to express the degree of restraint from the warping at the joint of elements 1 and 2. Equations (9) and (11) show that when $K=$ 1 , free warping occurs at end $x=0$ or $(y=-l)$, and fully restrained warping occurs when $K=0$.

The total SE $(U)$ of a SBLF with six-axis loads at free end was summarized as follows:

$$
U=U_{1}+U_{2},
$$

where $U_{1}$ is the total SE of element $1, U_{1}=U_{a 1}+U_{s 1}+U_{b 1}+U_{t 1}$; and $U_{2}$ is the total SE of element 2, $U_{2}=U_{a 2}+U_{s 2}+U_{b 2}+U_{t 2}$.

From (1), the translational displacements due to forces $F_{x}$, $F_{y}$, and $F_{z}$ according to the $x, y$, and $z$ axes, respectively, are given by

$$
\begin{aligned}
& \delta_{x}=\frac{\partial U}{\partial F_{x}}=\frac{F_{x} l}{A E}+\frac{F_{x} l t^{2}(1+\nu)}{2 E I_{z}}+\frac{1}{E I_{z}}\left(\frac{M_{z} l^{2}}{2}+\frac{F_{x} l^{3}}{3}+\frac{F_{y} l^{3}}{2}\right), \\
& \delta_{y}=\frac{\partial U}{\partial F_{y}}=\frac{F_{y} l}{A E}+\frac{F_{y} l t^{2}(1+\nu)}{2 E I_{z}}+\frac{1}{E I_{z}}\left(\frac{3 M_{z} l^{2}}{2}+\frac{4 F_{y} l^{3}}{3}+\frac{F_{x} l^{3}}{2}\right),
\end{aligned}
$$




$$
\begin{aligned}
\delta_{z}= & \frac{\partial U}{\partial F_{z}} \\
= & \frac{F_{z} l b^{2}(1+v)}{2 E I_{y}}-\frac{M_{z} l^{2}}{2 E I_{y}}+\frac{F_{z} l^{3}}{3 E I_{y}}-\frac{T_{x} l^{2}}{2 E I_{x}}+\frac{F_{z} l^{3}}{3 E I_{x}} \\
& +\frac{\left(F_{z} l^{2}-M_{y} l\right)\left(2 \alpha l-2 \sinh \alpha l+(2 \cosh \alpha l-\cosh 2 \alpha l-1) K_{t}+\alpha l K_{t}^{2}\right)}{\alpha G J_{y}} .
\end{aligned}
$$

The rotational displacements due to moments $T_{x}, M_{y}$, and $M_{z}$ about the $x, y$, and $z$ axes, respectively, are given by

$$
\begin{aligned}
& \theta_{x}=\frac{\partial U}{\partial T_{x}}=\frac{2 T_{x} l-F_{z} l^{2}}{2 E I_{x}}+\frac{T_{x}\left(K^{2} \sinh \alpha l-\sinh \alpha l+\alpha l \cosh \alpha l\right)}{\alpha G J_{x} \cosh \alpha l}, \\
& \theta_{y}=\frac{\partial U}{\partial M_{y}}=\frac{M_{y} l}{E I_{y}}-\frac{F_{z} l^{2}}{2 E I_{y}}+\frac{\left(M_{y}-F_{z} l\right)\left(2 \alpha l-2 \sinh \alpha l+(2 \cosh \alpha l-\cosh 2 \alpha l-1) K_{t}+\alpha l K_{t}^{2}\right)}{\alpha G J_{y}}, \\
& \theta_{z}=\frac{\partial U}{\partial M_{z}}=\frac{1}{E I_{z}}\left(2 M_{z} l+\frac{3 F_{y} l^{2}}{2}+\frac{F_{x} l^{2}}{2}\right),
\end{aligned}
$$

where

$$
K_{t}=\left(K \tanh \alpha l-(1-K)\left(\frac{1-\cosh \alpha l}{\sinh \alpha l}\right)\right) .
$$

2.2. Compliance Matrix of the SBLF. The relationship between the translational and rotational displacements and the applied forces and moments at the free end of the SBLF is formulated as follows:

$$
\begin{aligned}
\{\delta\} & =[C]\{f\}, \\
\{f\} & =[K]\{\delta\},
\end{aligned}
$$

where $\{\delta\}=\left[\delta_{x} \delta_{y} \delta_{z} \theta_{x} \theta_{y} \theta_{z}\right]^{T},\{f\}=\left[F_{x} F_{y} F_{z} T_{x} M_{y} M_{z}\right]^{T}$, $[C]$ is the compliance matrix or flexibility matrix, $[K]$ is the stiffness matrix, $F_{n}$ and $\delta_{n}$ are the force and translational displacement with respect to the $n$-axis, and $M_{n}$ and $\theta_{n}$ are the moment and rotational displacement about the $n$ axis, respectively. The compliance matrix $[C]$ includes 11 independent elements as follows:

$$
\begin{aligned}
& \left\{\begin{array}{l}
\delta_{x} \\
\delta_{y} \\
\delta_{z} \\
\theta_{x} \\
\theta_{y} \\
\theta_{z}
\end{array}\right\}=\left|\begin{array}{cccccc}
C_{\delta_{x} F_{x}} & C_{\delta_{x} F_{y}} & 0 & 0 & 0 & C_{\delta_{x} M_{z}} \\
C_{\delta_{y} F_{x}} & C_{\delta_{y} F_{y}} & 0 & 0 & 0 & C_{\delta_{y} M_{z}} \\
0 & 0 & C_{\delta_{z} F_{z}} & C_{\delta_{z} T_{x}} & C_{\delta_{z} M_{y}} & 0 \\
0 & 0 & C_{\theta_{x} F_{z}} & C_{\theta_{x} T_{x}} & 0 & 0 \\
0 & 0 & C_{\theta_{y} F_{z}} & 0 & C_{\theta_{y} M_{y}} & 0 \\
C_{\theta_{z} F_{x}} & C_{\theta_{z} F_{y}} & 0 & 0 & 0 & C_{\theta_{z} M_{z}}
\end{array}\right|\left\{\begin{array}{c}
F_{x} \\
F_{y} \\
F_{z} \\
T_{x} \\
M_{y} \\
M_{z}
\end{array}\right\}, \\
& C_{\delta_{x} F_{x}}=\left(\frac{\partial U}{\partial F_{x}}\right) \frac{1}{F_{x}}=\frac{l}{A E}+\frac{l t^{2}(1+\nu)}{4 E I_{z}}+\frac{l^{3}}{3 E I_{z}},
\end{aligned}
$$

$$
\begin{array}{rl}
C_{\delta_{x} F_{y}} & =\left(\frac{\partial U}{\partial F_{x}}\right) \frac{1}{F_{y}}=\frac{l^{3}}{2 E I_{z}}, \\
C_{\delta_{x} M_{z}} & =\left(\frac{\partial U}{\partial F_{x}}\right) \frac{1}{M_{z}}=\frac{l^{2}}{2 E I_{z}}, \\
C_{\delta_{y} F_{x}} & =\left(\frac{\partial U}{\partial F_{y}}\right) \frac{1}{F_{x}}=\frac{l^{3}}{2 E I_{z}}, \\
C_{\delta_{y} F_{y}} & =\left(\frac{\partial U}{\partial F_{y}}\right) \frac{1}{F_{y}}=\frac{l}{A E}+\frac{l t^{2}(1+\nu)}{4 E I_{z}}+\frac{4 l^{3}}{3 E I_{z}}, \\
C_{\delta_{y} M_{z}} & =\left(\frac{\partial U}{\partial F_{y}}\right) \frac{1}{M_{z}}=\frac{3 l^{3}}{2 E I_{z}}, \\
C_{\delta_{z} F_{z}} & =\left(\frac{\partial U}{\partial F_{z}}\right) \frac{1}{F_{z}}=\frac{l b^{2}(1+\nu)}{2 E I_{y}}+\frac{l^{3}}{3 E I_{y}}+\frac{l^{3}}{3 E I_{x}} \\
+l^{2} & \left(2 \alpha l-2 \sinh \alpha l+(2 \cosh \alpha l-\cosh 2 \alpha l-1) K_{t}+\alpha l K_{t}^{2}\right) \\
C_{\theta_{x} T_{x}} & =\left(\frac{\partial U}{\partial T_{x}}\right) \frac{1}{T_{x}}=\frac{l}{E I_{x}} \\
C_{\delta_{z} T_{x}} & =\left(\frac{\partial U}{\partial F_{z}}\right) \frac{1}{T_{x}}=-\frac{l^{2}}{2 E I_{x}}, \\
C_{\delta_{z} M_{y}} & =\left(\frac{\partial U}{\partial F_{z}}\right) \frac{1}{M_{y}}=-\frac{l^{2}}{2 E I_{y}} \\
l & l\left(2 \alpha l-2 \sinh \alpha l+(2 \cosh \alpha l-\cosh 2 \alpha l-1) K_{t}+\alpha l K_{t}^{2}\right) \\
F_{z} & =-\frac{l^{2}}{2 E I_{x}},
\end{array}
$$






(a) $\delta_{y}-l$



(b) $\delta_{y}-t$



(c) $\delta_{y}-b$

FIgURE 2: Variation of deflection $\delta_{y}$ according to (a) length $l$, (b) thickness $t$, and (c) width $b$ under $F_{y}$.

$$
\begin{aligned}
&+ \frac{\left(K^{2} \sinh \alpha l-\sinh \alpha l+\alpha l \cosh \alpha l\right)}{\alpha G J_{x} \cosh \alpha l}, \\
& C_{\theta_{y} F_{z}}=\left(\frac{\partial U}{\partial M_{y}}\right) \frac{1}{F_{z}}=-\frac{l^{2}}{2 E I_{y}} \\
&- \frac{l\left(2 \alpha l-2 \sinh \alpha l+(2 \cosh \alpha l-\cosh 2 \alpha l-1) K_{t}+\alpha l K_{t}^{2}\right)}{\alpha G J_{y}}, \\
& C_{\theta_{y} M_{y}}=\left(\frac{\partial U}{\partial M_{y}}\right) \frac{1}{M_{y}}=\frac{l}{E I_{y}} \\
&+\frac{l\left(2 \alpha l-2 \sinh \alpha l+(2 \cosh \alpha l-\cosh 2 \alpha l-1) K_{t}+\alpha l K_{t}^{2}\right)}{\alpha G J_{y}}, \\
& C_{\theta_{z} F_{x}}=\left(\frac{\partial U}{\partial M_{z}}\right) \frac{1}{F_{x}}=\frac{l^{2}}{2 E I_{z}},
\end{aligned}
$$$$
\begin{aligned}
& C_{\theta_{z} F_{y}}=\left(\frac{\partial U}{\partial M_{z}}\right) \frac{1}{F_{y}}=\frac{3 l^{3}}{2 E I_{z}}, \\
& C_{\theta_{z} M_{z}}=\left(\frac{\partial U}{\partial M_{z}}\right) \frac{1}{M_{z}}=\frac{2 l}{E I_{z}} .
\end{aligned}
$$

\section{FEA Verification}

In this study, FEA was conducted by using Pro-Mechanica commercial software (Wildfire 5, PTC Corp., MA, USA) to verify the results obtained by theory and to validate the reliability of the calculated results. The default values of the SBLF were chosen as follows: length $l=10 \mathrm{~mm}$, width $b=$ $4 \mathrm{~mm}$, and thickness $t=0.5 \mathrm{~mm}$. The forces and moments 
TABLE 1: Comparison between theory and the FEA at the default values of the SBLF.

\begin{tabular}{|c|c|c|c|c|c|}
\hline \multirow{2}{*}{ Displacements } & \multirow{2}{*}{ FEA } & \multicolumn{2}{|c|}{ Theory } & \multicolumn{2}{|c|}{ Error (\%) } \\
\hline & & Shear and Warp & No shear and No warp & $\mathrm{S}$ and $\mathrm{W}$ & No $S$ and No W \\
\hline$\overline{\delta_{x}(\mathrm{~mm})}$ & 1.16210 & 1.16466 & 1.16183 & 0.22 & -0.02 \\
\hline$\delta_{y}(\mathrm{~mm})$ & 0.44183 & 0.46480 & 0.46451 & 4.94 & 4.88 \\
\hline$\delta_{z}(\mathrm{~mm})$ & 0.21102 & 0.20825 & 0.22556 & 1.31 & 6.45 \\
\hline$\theta_{x}(\mathrm{rad})$ & 0.00239 & 0.00233 & 0.00251 & 2.19 & 5.04 \\
\hline$\theta_{y}(\mathrm{rad})$ & 0.00210 & 0.00209 & 0.00227 & 0.47 & 7.43 \\
\hline$\theta_{z}(\mathrm{rad})$ & 0.00673 & 0.00697 & 0.00697 & 3.43 & 3.43 \\
\hline
\end{tabular}

TABLE 2: Theoretical and FEA results with various lengths $l$, thicknesses $t$, and widths $b$ under $F_{x}, F_{y}$, and $F_{z}$.

\begin{tabular}{|c|c|c|c|c|c|c|c|c|c|c|c|}
\hline$l(\mathrm{~mm})$ & FEA & Theory & Error $(\%)$ & $t(\mathrm{~mm})$ & FEA & Theory & Error (\%) & $b(\mathrm{~mm})$ & FEA & Theory & Error (\%) \\
\hline \multicolumn{12}{|c|}{ (a) Deflection $\delta_{x}(\mathrm{~mm})$} \\
\hline 5 & 0.13819 & 0.14550 & 5.02 & 0.25 & 8.77255 & 9.29028 & 5.57 & 2 & 2.28306 & 2.32366 & 1.75 \\
\hline 6.5 & 0.30485 & 0.31934 & 4.54 & 0.325 & 3.97715 & 4.22907 & 5.96 & 2.6 & 1.74683 & 1.78743 & 2.27 \\
\hline 8 & 0.57071 & 0.59507 & 4.09 & 0.4 & 2.17394 & 2.26869 & 4.18 & 3.2 & 1.41120 & 1.45229 & 2.83 \\
\hline 9.5 & 0.95908 & 0.99619 & 3.72 & 0.475 & 1.30778 & 1.35502 & 3.49 & 3.8 & 1.18188 & 1.22298 & 3.36 \\
\hline 11 & 1.49324 & 1.54623 & 3.43 & 0.55 & 0.84862 & 0.87301 & 2.79 & 4.4 & 1.01548 & 1.05621 & 3.86 \\
\hline 12.5 & 2.19646 & 2.26869 & 3.18 & 0.625 & 0.58257 & 0.59507 & 2.10 & 5 & 0.88936 & 0.92946 & 4.32 \\
\hline 14 & 3.09203 & 3.18708 & 2.98 & 0.7 & 0.41772 & 0.42366 & 1.40 & 5.6 & 0.79058 & 0.82988 & 4.74 \\
\hline 15.5 & 4.20323 & 4.32493 & 2.81 & 0.775 & 0.31008 & 0.31227 & 0.70 & 6.2 & 0.71120 & 0.74957 & 5.12 \\
\hline 17 & 5.55339 & 5.70573 & 2.67 & 0.85 & 0.23676 & 0.23676 & 0.00 & 6.8 & 0.64607 & 0.68343 & 5.47 \\
\hline 18.5 & 7.16579 & 7.35301 & 2.55 & 0.925 & 0.18508 & 0.18377 & -0.71 & 7.4 & 0.59170 & 0.62802 & 5.78 \\
\hline 20 & 9.06374 & 9.29028 & 2.44 & 1 & 0.14756 & 0.14550 & -1.42 & 8 & 0.54565 & 0.58091 & 6.07 \\
\hline \multicolumn{12}{|c|}{ (b) Deflection $\delta_{y}(\mathrm{~mm})$} \\
\hline 5 & 0.05369 & 0.05809 & 7.58 & 0.25 & 3.55785 & 3.71567 & 4.25 & 2 & 0.90181 & 0.92903 & 2.93 \\
\hline 6.5 & 0.11959 & 0.12759 & 6.27 & 0.325 & 1.62113 & 1.69129 & 4.15 & 2.6 & 0.69073 & 0.71464 & 3.35 \\
\hline 8 & 0.22485 & 0.23785 & 5.47 & 0.4 & 0.86939 & 0.90720 & 4.17 & 3.2 & 0.55848 & 0.58064 & 3.82 \\
\hline 9.5 & 0.37879 & 0.39827 & 4.89 & 0.475 & 0.51931 & 0.54178 & 4.15 & 3.8 & 0.46800 & 0.48896 & 4.29 \\
\hline 11 & 0.59062 & 0.61825 & 4.47 & 0.55 & 0.33455 & 0.34901 & 4.14 & 4.4 & 0.40226 & 0.42229 & 4.74 \\
\hline 12.5 & 0.86949 & 0.90720 & 4.16 & 0.625 & 0.22798 & 0.23785 & 4.15 & 5 & 0.35238 & 0.37161 & 5.17 \\
\hline 14 & 1.22458 & 1.27453 & 3.92 & 0.7 & 0.16225 & 0.16931 & 4.17 & 5.6 & 0.31329 & 0.33180 & 5.58 \\
\hline 15.5 & 1.66503 & 1.72963 & 3.74 & 0.775 & 0.11953 & 0.12477 & 4.20 & 6.2 & 0.28185 & 0.29969 & 5.95 \\
\hline 17 & 2.19995 & 2.28192 & 3.59 & 0.85 & 0.09057 & 0.09458 & 4.24 & 6.8 & 0.25604 & 0.27324 & 6.29 \\
\hline 18.5 & 2.83849 & 2.94080 & 3.48 & 0.925 & 0.07025 & 0.07339 & 4.28 & 7.4 & 0.23449 & 0.25109 & 6.61 \\
\hline 20 & 3.58976 & 3.71567 & 3.39 & 1 & 0.05557 & 0.05809 & 4.33 & 8 & 0.21624 & 0.23226 & 6.90 \\
\hline \multicolumn{12}{|c|}{ (c) Deflection $\delta_{z}(\mathrm{~mm})$} \\
\hline 5 & 0.02203 & 0.02113 & 4.09 & 0.25 & 1.60255 & 1.58055 & 1.37 & 2 & 0.52645 & 0.51994 & 1.24 \\
\hline 6.5 & 0.05270 & 0.05127 & 2.72 & 0.325 & 0.74212 & 0.73006 & 1.63 & 2.6 & 0.37023 & 0.36566 & 1.24 \\
\hline 8 & 0.10341 & 0.10146 & 1.89 & 0.4 & 0.40469 & 0.39777 & 1.71 & 3.2 & 0.28218 & 0.27863 & 1.26 \\
\hline 9.5 & 0.17912 & 0.17672 & 1.34 & 0.475 & 0.24617 & 0.24151 & 1.90 & 3.8 & 0.22569 & 0.22270 & 1.33 \\
\hline 11 & 0.28479 & 0.28210 & 0.95 & 0.55 & 0.16164 & 0.15831 & 2.07 & 4.4 & 0.18640 & 0.18372 & 1.44 \\
\hline 12.5 & 0.42541 & 0.42263 & 0.65 & 0.625 & 0.11239 & 0.10988 & 2.24 & 5 & 0.15750 & 0.15497 & 1.61 \\
\hline 14 & 0.60595 & 0.60338 & 0.43 & 0.7 & 0.08169 & 0.07972 & 2.42 & 5.6 & 0.13538 & 0.13290 & 1.84 \\
\hline 15.5 & 0.83137 & 0.82938 & 0.24 & 0.775 & 0.06152 & 0.05992 & 2.61 & 6.2 & 0.11792 & 0.11542 & 2.12 \\
\hline 17 & 1.10665 & 1.10569 & 0.09 & 0.85 & 0.04770 & 0.04636 & 2.81 & 6.8 & 0.10379 & 0.10126 & 2.44 \\
\hline 18.5 & 1.43675 & 1.43737 & -0.04 & 0.925 & 0.03788 & 0.03675 & 3.01 & 7.4 & 0.09214 & 0.08956 & 2.81 \\
\hline 20 & 1.82663 & 1.82945 & -0.15 & 1 & 0.03071 & 0.02973 & 3.22 & 8 & 0.08239 & 0.07975 & 3.21 \\
\hline
\end{tabular}






(a) $\delta_{z}-l$



(b) $\delta_{z}-t$



(c) $\delta_{z}-b$

FIgURE 3: Variation of deflection $\delta_{z}$ according to (a) length $l$, (b) thickness $t$, and (c) width $b$ under $F_{z}$.

applied to the SBLF are $F_{x}=10 \mathrm{~N}, F_{y}=F_{z}=1 \mathrm{~N}$, and $T_{x}=$ $M_{y}=M_{z}=1 \mathrm{Nmm}$. The warping restraint factor was chosen to be $K=0.5$. The material selected in this simulation is aluminum $\mathrm{Al}$ 6061. In the parametric analysis, the sensitivity analysis was performed to determine the agreement between FEA and theory with the variation of length $l=5$ to $20 \mathrm{~mm}$, width $b=2$ to $8 \mathrm{~mm}$, and thickness $t=0.25$ to $1 \mathrm{~mm}$, respectively. If the errors between the two methods are lower than $10 \%$, the results can usually be accepted and used from the viewpoint of the engineering design.

Table 1 shows the comparison between the theory and FEA results at the default values of the SBLF. Equations (15)(16) were applied to find the translational and rotational displacements by the theoretical method that considers the variable shear deformation in the bending and the warping in both the bending and torsion and the partially restrained warping at the joint of elements 1 and 2 . The derived theoretical equations showed a good agreement with the FEA results; all of the errors of these simulations were less than $5 \%$, whereas the results of without shear and warp effect showed higher errors, up to $7.4 \%$ as presented in Table 1 . The results of in-plane deformations $\delta_{x}, \delta_{y}$, and $\theta_{z}$ in this study were consistent with results of the equations in [24]. Although these results were showing the good agreement at the default values, the sensitive parameters analysis was also conducted to ensure the reliability of the derived equations.

In the research of the reference [28], we analyzed the displacement of a SBLF under transverse loading by the Euler-Bernoulli, Timoshenko, and third-order beam theory and the results showed that the third-order beam theory of Levinson which considers the shear and warp effect in bending gave the good results. In this study, the above beam 
TABLE 3: Theoretical and FEA results with various lengths $l$, thicknesses $t$, and widths $b$ under loads $T_{x}, M_{y}$, and $M_{z}$.

\begin{tabular}{|c|c|c|c|c|c|c|c|c|c|c|c|}
\hline$l(\mathrm{~mm})$ & FEA & Theory & Error (\%) & $t(\mathrm{~mm})$ & FEA & Theory & Error (\%) & $b(\mathrm{~mm})$ & FEA & Theory & Error (\%) \\
\hline \multicolumn{12}{|c|}{ (a) Rotation $\theta_{x}(\mathrm{rad})$} \\
\hline 5 & 0.00114 & 0.00108 & 5.59 & 0.25 & 0.01795 & 0.01762 & 1.86 & 2 & 0.00568 & 0.00561 & 1.34 \\
\hline 6.5 & 0.00152 & 0.00145 & 4.04 & 0.325 & 0.00833 & 0.00815 & 2.15 & 2.6 & 0.00404 & 0.00397 & 1.66 \\
\hline 8 & 0.00189 & 0.00183 & 3.07 & 0.4 & 0.00455 & 0.00445 & 2.28 & 3.2 & 0.00312 & 0.00306 & 1.91 \\
\hline 9.5 & 0.00226 & 0.00221 & 2.40 & 0.475 & 0.00277 & 0.00270 & 2.50 & 3.8 & 0.00254 & 0.00248 & 2.13 \\
\hline 11 & 0.00263 & 0.00258 & 1.91 & 0.55 & 0.00183 & 0.00178 & 2.71 & 4.4 & 0.00213 & 0.00208 & 2.36 \\
\hline 12.5 & 0.00301 & 0.00296 & 1.53 & 0.625 & 0.00127 & 0.00123 & 2.89 & 5 & 0.00183 & 0.00179 & 2.61 \\
\hline 14 & 0.00338 & 0.00334 & 1.23 & 0.7 & 0.00093 & 0.00090 & 3.03 & 5.6 & 0.00161 & 0.00156 & 2.88 \\
\hline 15.5 & 0.00375 & 0.00371 & 0.99 & 0.775 & 0.00070 & 0.00068 & 3.16 & 6.2 & 0.00143 & 0.00138 & 3.18 \\
\hline 17 & 0.00412 & 0.00409 & 0.78 & 0.85 & 0.00054 & 0.00052 & 3.25 & 6.8 & 0.00128 & 0.00124 & 3.49 \\
\hline 18.5 & 0.00450 & 0.00447 & 0.60 & 0.925 & 0.00043 & 0.00042 & 3.33 & 7.4 & 0.00116 & 0.00112 & 3.83 \\
\hline 20 & 0.00487 & 0.00485 & 0.45 & 1 & 0.00035 & 0.00034 & 3.39 & 8 & 0.00106 & 0.00102 & 4.19 \\
\hline \multicolumn{12}{|c|}{ (b) Rotation $\theta_{y}(\mathrm{rad})$} \\
\hline 5 & 0.00089 & 0.00084 & 4.77 & 0.25 & 0.01605 & 0.01583 & 1.34 & 2 & 0.00540 & 0.00533 & 1.33 \\
\hline 6.5 & 0.00126 & 0.00122 & 3.08 & 0.325 & 0.00744 & 0.00732 & 1.60 & 2.6 & 0.00376 & 0.00371 & 1.35 \\
\hline 8 & 0.00163 & 0.00159 & 2.11 & 0.4 & 0.00407 & 0.00399 & 1.77 & 3.2 & 0.00285 & 0.00281 & 1.39 \\
\hline 9.5 & 0.00200 & 0.00197 & 1.49 & 0.475 & 0.00248 & 0.00243 & 2.01 & 3.8 & 0.00228 & 0.00224 & 1.46 \\
\hline 11 & 0.00237 & 0.00235 & 1.06 & 0.55 & 0.00163 & 0.00159 & 2.23 & 4.4 & 0.00188 & 0.00185 & 1.59 \\
\hline 12.5 & 0.00274 & 0.00272 & 0.74 & 0.625 & 0.00114 & 0.00111 & 2.46 & 5 & 0.00158 & 0.00155 & 1.77 \\
\hline 14 & 0.00312 & 0.00310 & 0.49 & 0.7 & 0.00083 & 0.00081 & 2.69 & 5.6 & 0.00136 & 0.00133 & 2.00 \\
\hline 15.5 & 0.00349 & 0.00348 & 0.29 & 0.775 & 0.00063 & 0.00061 & 2.92 & 6.2 & 0.00118 & 0.00116 & 2.29 \\
\hline 17 & 0.00386 & 0.00385 & 0.13 & 0.85 & 0.00049 & 0.00047 & 3.16 & 6.8 & 0.00104 & 0.00101 & 2.62 \\
\hline 18.5 & 0.00423 & 0.00423 & -0.01 & 0.925 & 0.00039 & 0.00037 & 3.41 & 7.4 & 0.00092 & 0.00090 & 2.99 \\
\hline 20 & 0.00460 & 0.00461 & -0.12 & 1 & 0.00032 & 0.00030 & 3.65 & 8 & 0.00082 & 0.00080 & 3.39 \\
\hline \multicolumn{12}{|c|}{ (c) Rotation $\theta_{z}(\mathrm{rad})$} \\
\hline 5 & 0.00328 & 0.00348 & 5.71 & 0.25 & 0.05405 & 0.05573 & 3.02 & 2 & 0.01365 & 0.01393 & 2.00 \\
\hline 6.5 & 0.00432 & 0.00453 & 4.70 & 0.325 & 0.02462 & 0.02537 & 2.94 & 2.6 & 0.01047 & 0.01072 & 2.31 \\
\hline 8 & 0.00535 & 0.00557 & 4.03 & 0.4 & 0.01320 & 0.01361 & 2.95 & 3.2 & 0.00848 & 0.00871 & 2.66 \\
\hline 9.5 & 0.00638 & 0.00662 & 3.58 & 0.475 & 0.00789 & 0.00813 & 2.94 & 3.8 & 0.00711 & 0.00733 & 3.01 \\
\hline 11 & 0.00741 & 0.00766 & 3.25 & 0.55 & 0.00508 & 0.00523 & 2.93 & 4.4 & 0.00612 & 0.00633 & 3.37 \\
\hline 12.5 & 0.00845 & 0.00871 & 3.02 & 0.625 & 0.00346 & 0.00357 & 2.93 & 5 & 0.00537 & 0.00557 & 3.72 \\
\hline 14 & 0.00948 & 0.00975 & 2.84 & 0.7 & 0.00246 & 0.00254 & 2.93 & 5.6 & 0.00477 & 0.00498 & 4.06 \\
\hline 15.5 & 0.01051 & 0.01080 & 2.71 & 0.775 & 0.00182 & 0.00187 & 2.94 & 6.2 & 0.00430 & 0.00449 & 4.39 \\
\hline 17 & 0.01153 & 0.01184 & 2.61 & 0.85 & 0.00138 & 0.00142 & 2.96 & 6.8 & 0.00391 & 0.00410 & 4.70 \\
\hline 18.5 & 0.01256 & 0.01289 & 2.53 & 0.925 & 0.00107 & 0.00110 & 2.98 & 7.4 & 0.00358 & 0.00377 & 5.01 \\
\hline 20 & 0.01359 & 0.01393 & 2.47 & 1 & 0.00084 & 0.00087 & 3.01 & 8 & 0.00330 & 0.00348 & 5.31 \\
\hline
\end{tabular}

theory was also used and the translational displacements $\delta_{x}$, $\delta_{y}$, and $\delta_{z}$ under $F_{x}, F_{y}$, and $F_{z}$ according to the variations of length $l$, thickness $t$, and width $b$, respectively, are presented in Table 2. Figure 2 shows typical simulation results under force $F_{y}$. The errors in these investigations are lower (7.6\%). The sensitive analysis of $F_{z}$ is also presented in Figure 3. All the errors are lower (5\%). These results reveal the accuracy of the derived theoretical equations for the translational displacements of SBLF.

The comparison results among the fully restrained, partially restrained, and free warping in torsion analysis of SBLF were investigated in [25] and the partially restrained warping with factor $K=0.5$ was applied in this torsion simulation. Table 3 shows the variation of rotational displacements $\theta_{x}, \theta_{y}$, and $\theta_{z}$ due to moments $T_{x}, M_{y}$, and $M_{z}$ with the variation of parameters $l, t$, and $b$ of the SBLF, respectively. It can be observed that the results of theory and FEA are in good agreement, with the errors lower than 6\%. Figure 4 presents the typical variation of rotation $\theta_{x}$ according to $l, t$, and $b$ under $T_{x}$. The maximum error is lower than $6 \%$.

In summary, the relationship between the deformation and the applied loads for the SBLF is presented in compliance matrix form. The variable shear deformation and warping effect were considered, and partial restraint was introduced in the analysis. All results were verified by FEA, with strong agreement between the two methods. The errors were lower than $7.6 \%$. These results demonstrate the high accuracy and reliability of the proposed theoretical equations. 




(a) $\theta_{x}-l$



(b) $\theta_{x}-t$



(c) $\theta_{x}-b$

Figure 4: Variation of rotation $\theta_{x}$ according to (a) length $l$, (b) thickness $t$, and (c) width $b$ under load $T_{x}$.

\section{Conclusion}

In this study, a compliance matrix that expresses the relationship between the deformations and applied loads of the SBLF was analyzed and derived by using Castigliano's second theorem. In bending analysis, higher-order beam theory was applied wherein variable shear deformation and the warping were considered in the calculated formulas for the shear forces. At the joint of two elements of the SBLF, the partially restrained warping in torsion was analysed with consideration of the warping restraint factor. The theoretical results were verified by FEA at both the default and the sensitive values. The results indicate that there is strong agreement between the two methods, with errors below
$7.6 \%$. This suggests the accuracy of the proposed theoretical equations and that they can be used in the precision machine design.

\section{Conflict of Interests}

The authors declare that there is no conflict of interests regarding the publication of this paper.

\section{Authors' Contribution}

Nghia-Huu Nguyen and Moo-Yeon Lee equally contributed to this work. 


\section{Acknowledgment}

This research was supported in part by Basic Science Research Program through the National Research Foundation of Korea (NRF) funded by the Ministry of Education (NRF2013R1A1A4A01009657).

\section{References}

[1] E.-J. Park, J. Shim, D.-Y. Lee, and J. Lee, "A double-bent planar leaf flexure guide for a nano-scanner," Journal of the Korean Physical Society, vol. 57, no. 61, pp. 1581-1588, 2010.

[2] K. Kim, D. Ahn, and D. Gweon, "Optimal design of a 1rotational DOF flexure joint for a 3-DOF H-type stage," Mechatronics, vol. 22, no. 1, pp. 24-32, 2011.

[3] T. T. Y. Koseki, T. Tanikawa, N. Koyachi, and T. Arai, "Kinematic analysis of a translational 3-d.o.f. micro-parallel mechanism using the matrix method," Advanced Robotics, vol. 16, no. 3, pp. 251-264, 2002.

[4] J. H. Kim, S. H. Kim, and Y. K. Kwak, "Development and optimization of 3-D bridge-type hinge mechanisms," Sensors and Actuators A: Physical, vol. 116, no. 3, pp. 530-538, 2004.

[5] J. W. Ryu, D.-G. Gweon, and K. S. Moon, "Optimal design of a flexure hinge based $X Y \varphi$ wafer stage," Precision Engineering, vol. 21, no. 1, pp. 18-28, 1997.

[6] N. Lobontiu and E. Garcia, "Analytical model of displacement amplification and stiffness optimization for a class of flexurebased compliant mechanisms," Computers \& Structures, vol. 81, no. 32, pp. 2797-2810, 2003.

[7] Y. $\mathrm{Li}$ and Q. Xu, "A novel piezoactuated XY stage with parallel, decoupled, and stacked flexure structure for micro/nanopositioning," IEEE Transactions on Industrial Electronics, vol. 58, no. 8, pp. 3601-3615, 2011.

[8] D. M. Brouwer, J. P. Meijaard, and J. B. Jonker, "Large deflection stiffness analysis of parallel prismatic leaf-spring flexures," Precision Engineering, vol. 37, no. 3, pp. 505-521, 2013.

[9] M. Hayashi and M. Fukuda, "Generation of nanometer displacement using reduction mechanism consisting of torsional leaf spring hinges," International Journal of Precision Engineering and Manufacturing, vol. 13, no. 5, pp. 679-684, 2012.

[10] J. J. Parise, L. L. Howell, and S. P. Magleby, "Ortho-planar linearmotion springs," Mechanism and Machine Theory, vol. 36, no. 11-12, pp. 1281-1299, 2001.

[11] S. Fukada and K. Nishimura, "Nanometric positioning over a one-millimeter stroke using a flexure guide and electromagnetic linear motor," International Journal of Precision Engineering and Manufacturing, vol. 8, pp. 49-53, 2007.

[12] Q. S. Xu, "Design and development of a compact flexure-based $\mathrm{XY}$ precision positioning system with centimeter range," IEEE Transactions on Industrial Electronics, vol. 61, no. 2, pp. 893-903, 2014.

[13] X.-P. S. Su and H. S. Yang, "Design of compliant microleverage mechanisms," Sensors and Actuators, A: Physical, vol. 87, no. 3, pp. 146-156, 2001.

[14] Y. K. Yong, S. S. Aphale, and S. O. R. Moheimani, "Design, identification, and control of a flexure-based XY stage for fast nanoscale positioning," IEEE Transactions on Nanotechnology, vol. 8, no. 1, pp. 46-54, 2009.

[15] J.-J. Kim, Y.-M. Choi, D. Ahn, B. Hwang, D.-G. Gweon, and J. Jeong, "A millimeter-range flexure-based nano-positioning stage using a self-guided displacement amplification mechanism," Mechanism and Machine Theory, vol. 50, pp. 109-120, 2012.

[16] S. Xiao, Y. Li, and Q. Meng, "Mobility analysis of a 3PUU flexure-based manipulator based on screw theory and compliance matrix method," International Journal of Precision Engineering and Manufacturing, vol. 14, no. 8, pp. 1345-1353, 2013.

[17] N. Lobontiu, "Compliance-based matrix method for modeling the quasi-static response of planar serial flexure-hinge mechanisms," Precision Engineering, vol. 38, no. 3, pp. 639-650, 2014.

[18] M. Kujawa, "Torsion of restrained thin-walled bars of open constraint bisymmetric cross-section," Tast Quarterly, vol. 16, pp. 5-15, 2011.

[19] E. J. Sapountzakis, "Bars under torsional loading: a generalized beam theory approach," ISRN Civil Engineering, vol. 2013, Article ID 916581, 39 pages, 2013.

[20] A. H. Al-HaKeem, Structural analysis of truck chassis frames under longitudinal loads considering bimoment effects [Ph.D. thesis], Cranfield Institute of Technology, Cranfield, UK, 1991.

[21] M. Levinson, "A new rectangular beam theory," Journal of Sound and Vibration, vol. 74, no. 1, pp. 81-87, 1981.

[22] J. N. Reddy, C. M. Wang, G. T. Lim, and K. H. Ng, "Bending solutions of Levinson beams and plates in terms of the classical Theories," International Journal of Solids and Structures, vol. 38, no. 26-27, pp. 4701-4720, 2001.

[23] C. M. Wang, J. N. Reddy, and K. H. Lee, Shear Deformable Beams and Plates: Relationships with Classical Solutions, Elsevier Science, Oxford, UK, 2000.

[24] N. Lobontiu and E. Garcia, Mechanics of Microelectromechanical Systems, Kluwer Academic, New York, NY, USA, 2005.

[25] N. H. Nguyen, B. D. Lim, and D. Y. Lee, “Torsional analysis of a single-bent leaf flexure," Structural Engineering and Mechanics, vol. 54, no. 1, pp. 189-198, 2015.

[26] S. P. Timoshenko and J. N. Goodier, Theory of Elasticity, McGraw-Hill, 2nd edition, 1951.

[27] W. D. Pilkey, Analysis and Design of Elastic Beams: Computational Methods, John Wiley \& Sons, New York, NY, USA, 2002.

[28] N. H. Nguyen, B. D. Lim, and D. Y. Lee, "Displacement analysis of a single-bent leaf flexure under transverse load," International Journal of Precision Engineering and Manufacturing, vol. 16, no. 4, pp. 749-754, 2015. 





The Scientific World Journal


Submit your manuscripts at http://www.hindawi.com
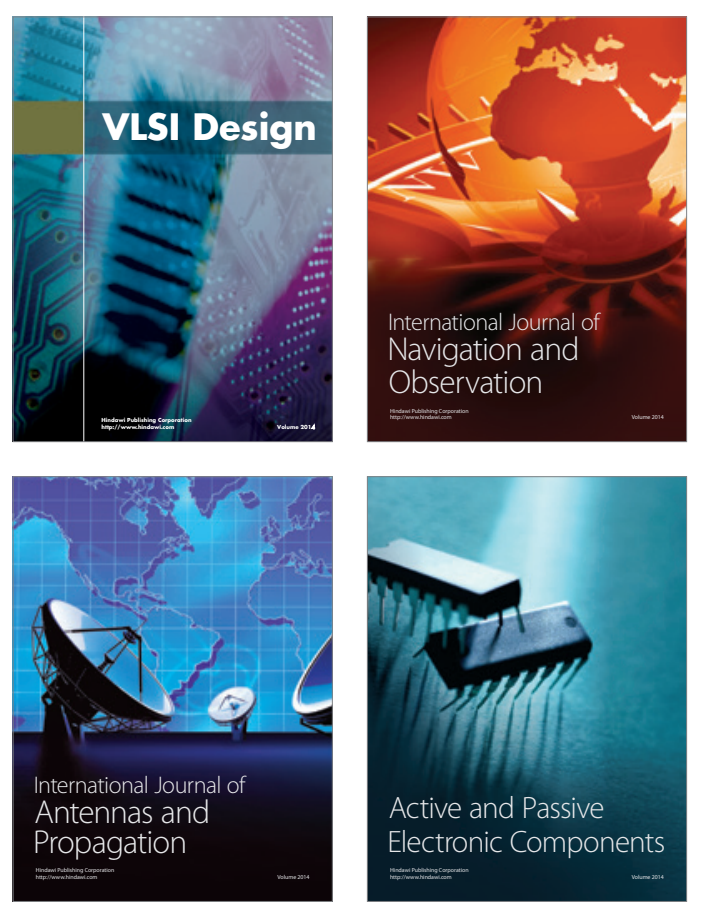
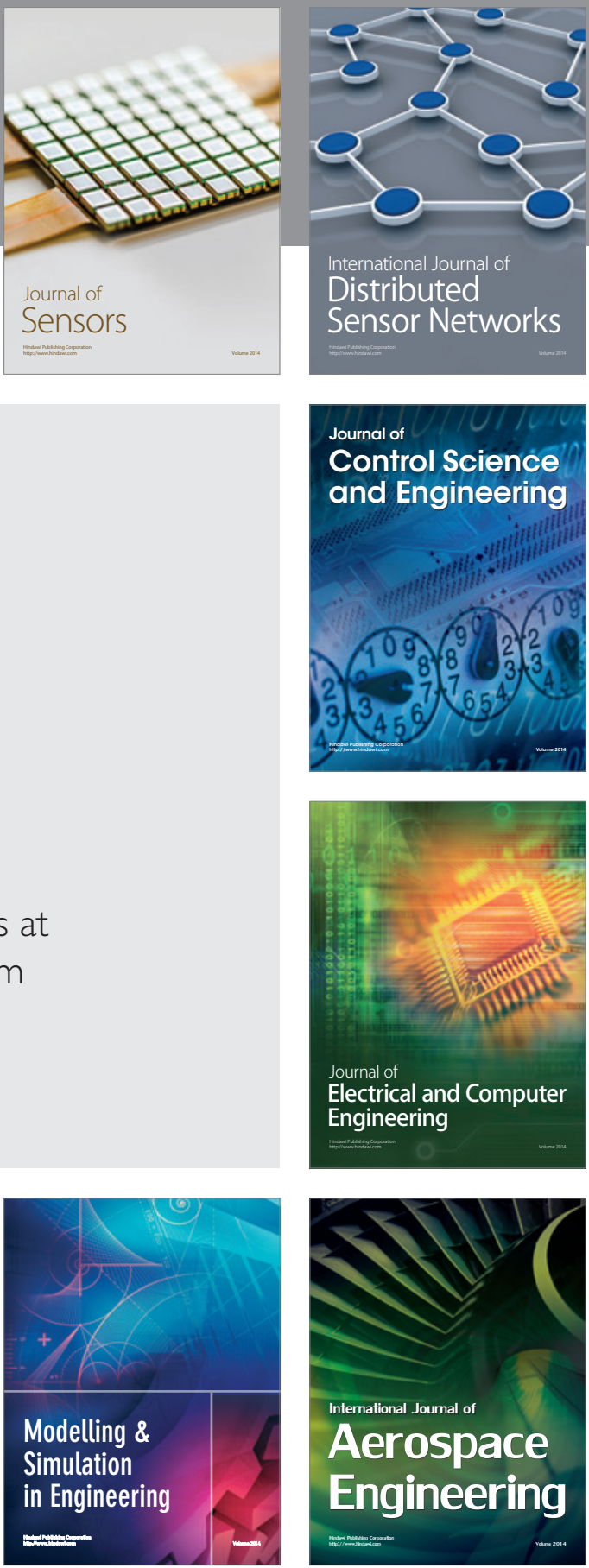

Journal of

Control Science

and Engineering
